\title{
Free caspase activity in CSF of patients with dementia
}

\author{
P. Albrecht - M. Schmitz $\cdot$ M. Otto $\cdot$ B. Hemmer · \\ I. Zerr $\cdot$ H.-P. Hartung $\cdot$ Axel Methner
}

Received: 11 December 2008/Revised: 4 January 2009/Accepted: 17 April 2009/Published online: 8 May 2009

(c) Springer-Verlag 2009

Sirs,

Neurodegenerative dementias such as sporadic Creutzfeldt-Jakob disease (sCJD), Alzheimer's disease (AD), and dementia with Lewy bodies (DLB) are difficult to diagnose. Quantification of brain-derived proteins, 14-3-3 in $\mathrm{sCJD}$, and tau and amyloid- $\beta$ in $\mathrm{AD}$, can support the clinical diagnosis, but results vary considerably [3, 6]. Caspase-mediated apoptosis was reported to be involved in the pathogenesis of SCJD [4] and appears to play a role in later stages of $\mathrm{AD}$ and DLB (reviewed in [5]). We hypothesized that the activity of effector caspases- 3 and -7 might be a sensitive indicator of apoptotic activity and potentially indicative of disease severity. Previous studies on the role of apoptosis in post mortem tissue yielded contradictory results, probably due to the fact that cell death in these illnesses occurs over decades, whereas the suicide program in the single cell is executed within a few hours. Thus, synchronous detection of a substantial number of apoptotic neurons seems almost impossible [5]. Cell-free

P. Albrecht · H.-P. Hartung · A. Methner $(\bowtie)$

Department of Neurology,

Heinrich-Heine Universität Düsseldorf,

Moorenstr. 5, 40225 Düsseldorf, Germany

e-mail: axel.methner@gmail.com

M. Schmitz $\cdot$ I. Zerr

Department of Neurology, University Medical School, Georg-August University of Göttingen, Göttingen, Germany

M. Otto

Department of Neurology, University Clinic of Ulm, Ulm, Germany

B. Hemmer

Department of Neurology, Klinikum rechts der Isar,

Technical University, Munich, Germany caspase activity in contrast is stable over a significant period of time and can be measured in serum, where it correlates with hepatic degeneration in patients with chronic hepatitis [7], and in cerebrospinal fluid (CSF), where it correlates with intracranial pressure in patients with head trauma [8].

In this contribution, we evaluated the use of free caspase activity in CSF as a marker for the activity of apoptosis in neurodegenerative dementias by measuring caspase-3/7 activity in CSF from patients with $\operatorname{sCJD}(n=13), \mathrm{AD}$ $(n=9)$, DLB $(n=7)$, and controls $(n=8)$. We correlated caspase activity with clinical status, course of disease, and established paraclinical tests.

$\mathrm{AD}$ and DLB was diagnosed according to DSM-IV and SCJD to WHO 98. Three SCJD patients were tapped consecutively for diagnostic reasons. Controls were two patients with isolated cervical dystonia, one with pseudotumor cerebri, and five without neurological disease, who gave informed consent. Clinical status was scored using the modified Rankin scale [1] and mini-mental-state examination (MMSE) [2]. Caspase activity was measured using Caspase-Glo 3/7 (Promega) and normalized with purified caspase-3 (Biomol). Protein 14-3-3 immunoblots and tau and amyloid- $\beta$ 1-42 enzyme-linked immunosorbent assay (ELISA) were performed as described [3, 4].

Mean caspase activity in CSF was 1,377 U/l (standard error of the mean, SEM 434.6 U/l) for sCJD patients, $338 \mathrm{U} / 1$ (SEM $84.8 \mathrm{U} / \mathrm{l}$ ) for AD patients, and $222 \mathrm{U} / 1$ (SEM 15.7 U/l) for DLB patients; for controls it was $215.1 \mathrm{U} / 1$ (SEM 24.3 U/1) (Fig. 1a). Only sCJD patients differed significantly from controls $(P<0.05$, two-tailed $t$ test; no repeated measurements were used for statistical analysis). In two patients with damage to the blood-brain barrier indicated by increased albumin quotient $\left(\mathrm{Q}_{\mathrm{ALB}} 10.5\right.$ and 11.5), caspase activity was higher in CSF, suggesting 


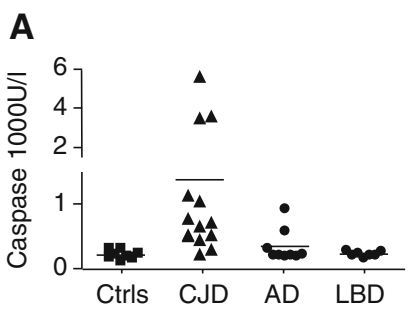

B
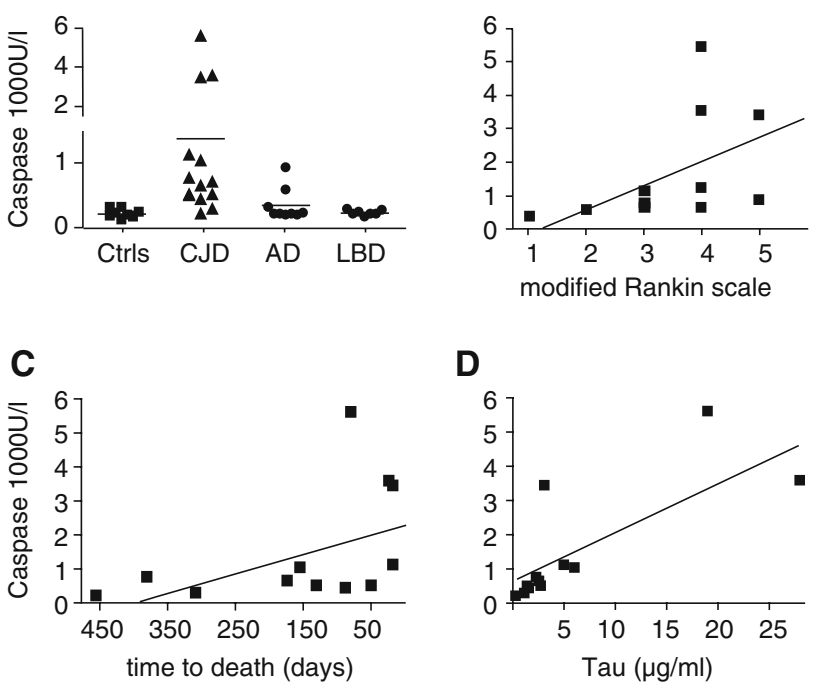

\section{D}

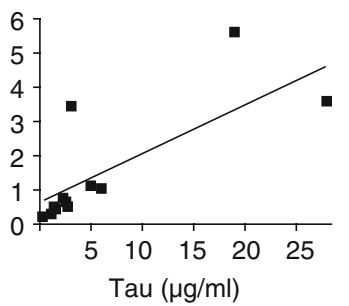

death, protein tau levels, and clinical status only in SCJD. Caspase activity cannot distinguish AD/DLB from sCJD but favors a diagnosis of sCJD and suggests a highly active disease, as rapidly deteriorating patients had higher caspase levels. Studies on a larger collective are underway to confirm the value of this novel marker of disease activity.

Fig. 1 a Dot plot of free caspase-3 and -7 activity in CSF from 13 sCJD patients, 9 AD patients, 7 DLB patients, and 8 controls, b caspase activity over clinical status and c remaining lifespan after spinal tap in SCJD-patients, $\mathbf{d}$ correlation of caspase- 3 and -7 activity with protein tau levels in CSF of sCJD patients

an origin within the nervous system. Elevated caspase activity correlated with clinical disability $(r=0.670$, $P=0.020$, Spearman, Fig. 1b), remaining lifespan ( $r=0.648, P=0.025$, Spearman, Fig. 1c), and levels of protein tau ( $r=0.779, P=0.003$, Pearson, Fig. 1d) only in patients with SCJD. In AD and DLB, caspase activity did not correlate with disease duration or MMSE. We observed no correlation of caspase activity with levels of amyloid- $\beta$ or of protein tau with Rankin scale. In consecutively tapped patients, free caspase levels and clinical disability increased over time (not shown).

We conclude that cell-free caspase activity can be measured in CSF and is significantly increased in SCJD, but not in AD or DLB. Caspase activity correlated with time to

\section{References}

1. Bonita R, Beaglehole R (1988) Recovery of motor function after stroke. Stroke 19:1497-1500

2. Folstein MF, Folstein SE, McHugh PR (1975) Mini-mental state. A practical method for grading the cognitive state of patients for the clinician. J Psychiatr Res 12:189-198. doi:10.1016/00223956(75)90026-6

3. Gloeckner SF, Meyne F, Wagner F, Heinemann U, Krasnianski A, Meissner B, Zerr I (2008) Quantitative analysis of transthyretin, tau and amyloid-beta in patients with dementia. J Alzheimers Dis $14: 17-25$

4. Hetz C, Russelakis-Carneiro M, Maundrell K, Castilla J, Soto C (2003) Caspase-12 and endoplasmic reticulum stress mediate neurotoxicity of pathological prion protein. EMBO J 22:54355445. doi:10.1093/emboj/cdg537

5. Kermer P, Liman J, Weishaupt JH, Bahr M (2004) Neuronal apoptosis in neurodegenerative diseases: from basic research to clinical application. Neurodegener Dis 1:9-19. doi:10.1159/ 000076665

6. Otto M, Wiltfang J, Cepek L, Neumann M, Mollenhauer B, Steinacker P, Ciesielczyk B, Schulz-Schaeffer W, Kretzschmar HA, Poser S (2002) Tau protein and 14-3-3 protein in the differential diagnosis of Creutzfeldt-Jakob disease. Neurology 58:192-197

7. Seidel N, Volkmann X, Langer F, Flemming P, Manns MP, Schulze-Osthoff K, Bantel H (2005) The extent of liver steatosis in chronic hepatitis $\mathrm{C}$ virus infection is mirrored by caspase activity in serum. Hepatology 42:113-120. doi:10.1002/hep.20747

8. Uzan M, Erman H, Tanriverdi T, Sanus GZ, Kafadar A, Uzun H (2006) Evaluation of apoptosis in cerebrospinal fluid of patients with severe head injury. Acta Neurochir (Wien) 148:1157-1164. doi:10.1007/s00701-006-0887-1 discussion 